\title{
hVISA/VISA: diagnostic and therapeutic problems
}

Expert Rev. Anti Infect. Ther. 7(1), 1-3 (2009)

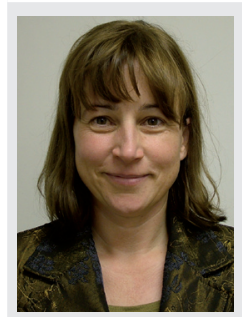

Judit Szabó

Department of Medical Microbiology, Medical and Health Science Center, University of Debrecen, P.O.B. 17, H-4012 Debrecen, Hungary

Tel.: + 3652414948 Fax: +3652414948 szabjud@dote.hu

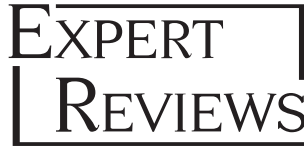

\author{
"Genetic analysis of vancomycin-intermediate Staphylococcus aureus \\ strains has provided further evidence that vancomycin resistance \\ develops due to changes of several loss-of-function mutations..."
}

Vancomycin is considered the goldstandard treatment for infections caused by methicillin-resistant Staphylococcus aureus (MRSA). In recent years, reduced susceptibility of $S$. aureus to vancomycin has emerged. This problem seems to be based on the increased use of vancomycin, with characteristic poor tissue penetration, slow bactericidal activity and risk of nephrotoxicity resulting lack of elimination of MRSA from the patient [1].

Vancomycin-intermediate $S$. aureus and heterogeneous vancomycin-intermediate $S$. aureus According to the Clinical and Laboratory Standards Institute (CLSI), vancomycinintermediate $S$. aureus (VISA) are those isolates with a MIC between 4 and $8 \mathrm{mg} / \mathrm{l}$, whereas heterogeneous VISA (hVISA) strains appear to be sensitive to vancomycin with susceptible range of 1-2 mg/l, but containing subpopulation of vancomycin-intermediate daughter cells (MIC $\geq 4 \mu \mathrm{g} / \mathrm{ml}$ ). Vancomycin-resistant $S$. aureus (VRSA) are defined as those having MICs of at least $16 \mathrm{mg} / \mathrm{l}$ [2].

\section{"Humans are the main reservoir of hVISA/VISA strains, which are capable of colonizing the environment and persisting, despite repeated and concerted eradication efforts."}

The first hVISA strain was isolated in Japan in 1996 [3]. Since 1996, hVISA and VISA strains have increased in Europe, Asia and the USA, including over 100 cases [4]. VRSA strains are still rare, and the first case was documented in 2002 .
As the VISA/VRSA isolates are also resistant to teicoplanin, the term glycopeptide-intermediate or glycopeptideresistant $S$. aureus is a technically more accurate term; however, the term VISA/VRSA is more widely used. In the USA, where teicoplanin is not available, the term VISA/VRSA is routinely applied [5].

Patients most at risk of infection by hVISA, VISA and VRSA appear to be those with previous exposure to vancomycin. Treatment options for infections due to MRSA with reduced susceptibility to vancomycin are limited. Rapid and correct identification of patients harboring VISA, hVISA and VRSA, as well as the prompt infection-control protocols, are very important in controlling the dissemination and selection of these strains [1].

\section{Mechanism of resistance of hVISA/VISA}

Strains of VISA have been observed to have lower growth rates and thicker cell walls than susceptible strains [4]. More murein monomers and more layers of peptidoglycan are considered to be present in the cell wall of VISA strains [3].

Genetic analysis of VISA strains has provided further evidence that vancomycin resistance develops due to changes of several loss-of-function mutations affecting important cell wall biosynthesis and intermediary metabolism genes [6].

The agr operon in $S$. aureus coordinates many critical virulence pathways. Activation of the agr operon induces the production of secreted virulence factors, such as hemolysins, exoproteins and exotoxins, and decreases the production of cell-associated virulence factors, such as 
adhesins. Expression of agr is inversely related to fibronectinbinding protein production, which offers selective advantage for the adherence to biomedical devices (e.g., catheters) and nasal colonization of patients or staff members. Under vancomycin exposure, loss of function of agr provides the selection of hVISA/VISA strains [7].

As a result of the loss of agr function:

- Biofilm production increases

- Autolysis due to the expression of murein hydrolase decreasing

- The activity of $\delta$-hemolysin either decreases or stops completely

- Cell wall synthesis changes (thicker cell wall)

\section{Laboratory diagnosis of VISA \& hVISA strains}

Traditionally, the agar disk-diffusion test has been used to measure glycopeptide susceptibility, but this method is not suitable for large molecules, such as vancomycin, because the diffusion of this antibiotic into agar is too slow [8].

An incorrect classification of MRSA as sensitive to glycopeptide antibiotics may occur, since glycopeptide MICs are dependent on test conditions. To date, no standardized technique for identifying hVISA strains has been established [4].

A variety of different screening plates have been described. Some studies were screened with Mueller-Hinton agar instead of brain-heart infusion (BHI) agar. Others applied a different inoculum size (100 vs $10 \mu \mathrm{l})$, while others used different concentration of bacterial suspension (2.0 vs $0.5 \mathrm{McFarland}$ ). Several studies screened with plates containing vanomycin 6,5 or $4 \mu \mathrm{g}$ (or teicoplanin) per $\mathrm{ml}$ [4]. The sensitivity was varied between 58 and $98 \%$, while the specificity was observed between 68 and 97\%. The most suitable screening method seems to be the Mueller-Hinton agar plate containing teicoplanin $5 \mu \mathrm{g}$ with $10 \mu \mathrm{l}$ of $2.0 \mathrm{McF}$ arland bacterial suspension incubated for $48 \mathrm{~h}$ [9].

"To date, no standardized technique for identifying hVISA strains has been established..."

Acceptable methods used to detect VISA/hVISA are nonautomated. The most precise method of determination of heteroresistance is a population-analysis profile, as follows. After $24 \mathrm{~h}$ incubation in BHI, cultures were diluted in saline to $10^{-3}$ and $10^{-6}$, and spiral plated on to $\mathrm{BHI}$ agar plates containing vancomycin $0.5,1$, 2, 2.5 and $4 \mathrm{mg} / \mathrm{l}$. Colonies were counted after $48 \mathrm{~h}$ incubation at $37^{\circ} \mathrm{C}$ and the viable count was used to calculate an AUC. To distinguish VISA, hVISA and vancomycin-sensitive $S$. aureus, a ratio of the AUC of test strains divided by the corresponding AUC for control strain was calculated. The criteria used for detection of hVISA and VISA were AUC ratios of at least 0.9 and at least 1.3, respectively [10]. The routine use of this method in a laboratory is time consuming and expensive; therefore, there is an urgent need for a simpler laboratory screening technique.

The macro E-test ${ }^{\circledR}$ method may be one possible way to identify true hVISA strains under routine laboratory conditions. The strains are grown overnight to a $2.0 \mathrm{McF}$ arland standard in
Mueller-Hinton broth. A 200- $\mu$ l sample is plated onto a BHI agar, and vancomycin and teicoplanin E-test ${ }^{\circledR}$ strips are applied thereafter. Plates are incubated for $48 \mathrm{~h}$ and then evaluated for growth according to the CLSI. The newest available double-sided strip is the combined vancomycin and teicoplanin strip, known as the glycopeptide-resistance detection strip. This E-test is performed on Mueller-Hinton plates, rather then BHI. The inoculum is $0.5 \mathrm{McF}$ arland and the incubation period is $48 \mathrm{~h}$. The cut-off values after $48 \mathrm{~h}$ were teicoplanin of at least $12 \mathrm{mg} / \mathrm{l}$, or both teicoplanin and vancomycin of at least $8 \mathrm{mg} / \mathrm{l}$. The standard vancomycin MIC is at least $6 \mathrm{mg} / \mathrm{l}$ for VISA and at least $4 \mathrm{mg} / \mathrm{l}$ for hVISA. The specificity and the sensitivity of the glycopeptide-resistance detection test is 94 and $95 \%$, respectively [9].

\section{Treatment of hVISA/VISA cases}

The hVISA/VISA infections are usually associated with poorer patient outcomes. The mortality rate for hVISA/VISA patients is approximately $75 \%[6]$.

Heterogeneous VISA seems to be the stage that precedes the development of VISA, therefore, when the vancomycin MIC is greater than $1 \mathrm{mg} / \mathrm{l}$, alternative therapies should be considered to avoid the possibility of treatment failure and selection of hVISA/VISA strains.

\section{${ }^{66}$ Currently, there are no formal recommendations regarding treatment of hVISA/VISA, and the future role of experimental and available antibiotics is unknown."}

Currently available drugs with activity against hVISA/VISA strains are linezolid, quinupristin/dalfopristin, trimethoprim/ sulfamethoxazole, the lipopeptide daptomycin and the tetracycline derivative tigecycline. Of these, trimethoprim/sulfamethoxazole and daptomycin are bactericidal. Trimethoprim/sulfamethoxazole is recommended in therapy only for skin and soft-tissue infections caused by hVISA/VISA strains. Experimental drugs with bactericidal activity against hVISA/VISA isolates include the lipoglycopeptide dalbavancin, the semisynthetic glycopeptide oritavancin, the glycolipodepsipeptide ramoplanin and a new broadspectrum cephalosporin, ceftobiprole. The in vitro activities of these drugs have been examined, although the clinical efficacy has not yet been established.

Currently, there are no formal recommendations regarding treatment of hVISA/VISA, and the future role of experimental and available antibiotics is unknown [6].

\section{Control of dissemination of hVISA/VISA strains}

Humans are the main reservoir of hVISA/VISA strains, which are capable of colonizing the environment and persisting, despite repeated and concerted eradication efforts. Fundamental hygiene habits are the primary defense against dissemination of the bacteria, including wearing a gown on entry into the patient's room, use of an alcohol rub when washing hands on exiting the room, one-to-one nursing and routine contact investigation while the 
patient is in hospital. Active communication between the clinician and the microbiology laboratory is essential if these strains are not to be missed.

Early, appropriate detection of resistance, prudent use of antibiotics, including vancomycin, and stringent infection control procedures may all be critical to controlling of infection and colonization of hVISA/VISA strains [6].

\section{Financial \& competing interests disclosure}

The author has no relevant affiliations or financial involvement with any organization or entity with a financial interest in or financial conflict with the subject matter or materials discussed in the manuscript. This includes employment, consultancies, honoraria, stock ownership or options, expert testimony, grants or patents received or pending, or royalties.

No writing assistance was utilized in the production of this manuscript.

\section{References}

1 Hiramatsu K. Vancomycin-resistant Staphylococcus aureus: a new model of antibiotic resistance. Lancet 1, 147-155 (2001).

2 Clinical and Laboratory Standards Institute. Performance standards for antimicrobial susceptibility testing: eighteenth international supplement M100-S18. Clinical and Laboratory Standards Institute, Wayne, PA, USA (2008).

3 Hiramatsu K, Aritaka N, Hanaki S et al. Dissemination in Japanese hospitals of strains of Staphylococcus aureus heterogeneously resistant to vancomycin. Lancet 350, 1670-1673 (1997).

4 Liu C and Chambers FH. Staphylococcus aureus with heterogeous resistance to vancomycin: epidemiology, clinical significance and critical assessment of diagnostic methods. Antimicrob. Agents Chemother. 47, 3040-3045 (2003).
5 Gould IM. Clinical relevance of increasing glycopeptides MICs against Staphylococcus aureus. Int. J. Antimicrob. Agents 31, 1-9 (2008).

6 Appelbaum PC. Reduced glycopeptide susceptibility in methicillin-resistant Staphylococcus aureus (MRSA). Int. J. Antimicrob. Agents 30, 398-408 (2007).

7 Sakoulas G, Moellering RC Jr, Eliopoulos GM. Adaptation of methicillin-resistant Staphylococcus aureus in the face of vancomycin therapy. Clin. Infect. Dis. 42, S40-S50 (2006).

8 Gould IM. The problem with glycopeptides. Int. J. Antimicrob. Agents 30, 1-3 (2007).

9 Wootton M, Howe RA, Hillman R et al. A modified population analysis profile (PAP) method to detect hetero-resistance to vancomycin in Staphylococcus aureus in a UK hospital. J. Antimicrob. Chemother. 47, 399-404 (2001).
10 Yusof A, Engelhardt A, Karlsson A et al. Evaluation of a new Etest vancomycinteicoplanin strip for detection of glycopeptide-intermediate Staphylococcus aureus (GISA), in particular, heterogeneous GISA. J. Clin. Microbiol. 46(9), 3042-3047 (2008).

\section{Affiliation}

- Judit Szabó, MD, PhD

Department of Medical Microbiology, Medical and Health Science Center, University of Debrecen, P.O.B. 17, H-4012 Debrecen, Hungary Tel.: +36 52414948

Fax: +36 52414948

szabjud@dote.hu 